\title{
Chiral dynamics with (non)strange quarks
}

\author{
Bastian Kubis ${ }^{1, \star}$ and Ulf-G. Meißner ${ }^{1,2, \star \star}$ \\ ${ }^{1}$ Universität Bonn, Helmholtz Institut für Strahlen- und Kernphysik, Bethe Center for Theoretical Physics, D-53115 Bonn, Germany \\ ${ }^{2}$ Forschungszentrum Jülich, Institute für Kernphysik (IKP-3), Institute for Advanced Simulation (IAS-4) and Jülich Center for \\ Hadron Physics, D-52145 Jülich, Germany
}

\begin{abstract}
We review the results and achievements of the project B.3. Topics addressed include pion photoproduction off the proton and off deuterium, three-flavor chiral perturbation theory studies, chiral symmetry tests in Goldstone boson decays, the development of unitarized chiral perturbation theory to next-to-leading order, the two-pole structure of the $\Lambda(1405)$, the dynamical generation of the lowest $S_{11}$ resonances, the theory of hadronic atoms and its application to various systems, precision studies in light-meson decays based on dispersion theory, the Roy-Steiner analysis of pion-nucleon scattering, a high-precision extraction of the elusive pion-nucleon $\sigma$-term, and aspects of chiral dynamics in few-nucleon systems.
\end{abstract}

\section{Introduction}

The project B.3 has been funded over the whole period of the SFB/TR 16. In the first funding period, it actually focused on "Meson production off nucleons and light nuclei" (title in the first funding period), spanning chiral perturbation theory (ChPT) calculations in the single-nucleon sector, extensions of three-flavor ChPT to higher energies by unitarization methods, and developments of chiral nuclear effective field theory (EFT) for light nuclei, with an emphasis on meson production reactions. The PIs in this funding period were Ulf Meißner and Akaki Rusetsky. As this project was extremely successful but also very broad, it was decided to move the nuclear aspects into the new project B.6 "Neutron properties from light nuclei" in funding periods 2 and 3, with the PIs Evgeny Epelbaum and Hans-Werner Hammer (and Ulf Meißner after 2013 when Hans-Werner Hammer moved to Darmstadt). In the second and third funding period, the project focused on precision calculations in meson decays, largely in the framework of dispersion relations, on the further development of unitarized ChPT for meson-baryon interactions, and on Roy-Steiner equations for pion-nucleon scattering. The advances made in these fields will be reported in chronological order, grouped into the three four-year funding periods.

Our contribution is organized as follows: Section 2 summarize the achievements from the first funding period. In the second funding period, Sec. 3 the research was more focused on Goldstone boson decays, Sec. 3.1, and on chiral coupled-channel analysis of meson-baryon scattering and meson production, Sec. 3.2. In the third funding period, Sec. 4, we worked on precision studies in light-meson

\footnotetext{
^e-mail: kubis@hiskp.uni-bonn.de

${ }^{\star}$ e-mail: meissner@hiskp.uni-bonn.de
}

decays, Sec. 4.1, revisited (anti)kaon-nucleon scattering, see Sec. 4.2, analyzed the $\Lambda(1405)$ in kaon photoproduction, see Sec. 4.3, and, last but not least, performed a detailed Roy-Steiner analysis of pion-nucleon scattering, see Sec. 4.4. In fact, the foundation of the Roy-Steiner studies were already laid out in the second funding period, but we prefer to report these topics in one section. Finally, an important disclaimer: This is the review of the project B.3, but not a general review of the various fields to which this research contributed. Therefore, only the papers that resulted from this research are cited, with the exception of only a few external papers and of course, in case data were involved, with proper reference to the experimental papers. The links to the other projects are mentioned in passing, but no effort is made to discuss these in any detail.

\section{First funding period 2004-2008}

\subsection{Pion photoproduction}

Neutral pion photoproduction off nucleons is one of the best reactions to test two-flavor baryon chiral dynamics. In Ref. [1] we have analyzed the Fubini-Furlan-Rosetti sum rule (which was derived in 1965 using soft-pion techniques) in the framework of covariant baryon chiral perturbation theory to leading one-loop accuracy and including next-to-leading order (NLO) polynomial contributions. We have shown how the appearing subtraction constants in the invariant amplitudes can be mapped onto certain low-energy constants (LECs) employed in earlier ChPT studies of threshold neutral pion photoproduction off nucleons. In particular, we consider the corrections to the sum rule due to the finite pion mass and show that below the threshold, they agree well with determinations based on fixed- $t$ dispersion relations performed by 


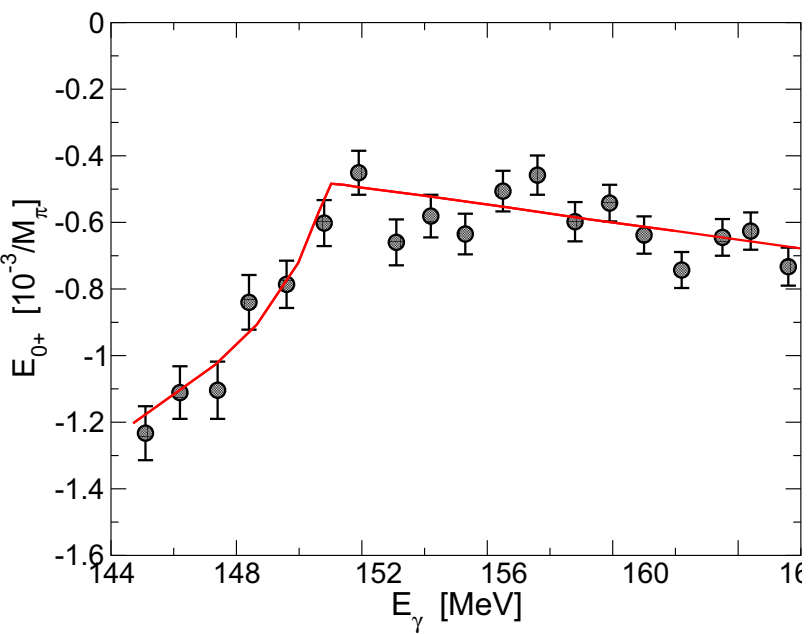

Figure 1. The real part of the electric dipole amplitude $E_{0+}$ in the threshold region. The solid line is the theoretical prediction as discussed in the text and the data are from Ref. [2]. The cusp at the opening of the $\pi^{+} n$ threshold $\left(E_{\gamma}=151.8 \mathrm{MeV}\right)$ is clearly visible. Figure taken from Ref. [1].

the Mainz group. We also have shown that this representation allows for a precise determination of certain LECs and we have given the most accurate $\mathrm{ChPT}$ prediction for the energy dependence of the electric dipole amplitude $E_{0+}$ in the threshold region, see Fig. 1.

\subsection{Three-flavor ChPT studies}

To make further progress in SU(3) chiral dynamics, we have also studied the application of ChPT in a variety of kaon decays of current interest because high-precision data emerge in particular from NA 48/2 at CERN. Aspects of radiative kaon decays were studied in Refs. [3-5], with particular emphasis on the possibility to extract structuredependent terms and the role of the axial anomaly. In Ref. [6], an effective field theory framework was formulated that allows one to extract the pion-pion S-wave scattering lengths from the structure of the cusp in the reaction $K \rightarrow 3 \pi$, originating from the $\pi^{0} \pi^{0}$ rescattering in the final state (that was earlier pointed out by Meißner, Müller and Steininger [7] a decade ago). We have also investigated the scalar sector of QCD as tested in $J / \psi$ decays to vector mesons and two pseudoscalars [8] and extracted the large- $N_{c}$-suppressed low-energy constant $L_{4}$ and $L_{6}$ of the fourth-order ChPT Lagrangian. This analysis was extended in Ref. [9] to include isospin-breaking terms in order to investigate the phenomenon of $a_{0}(980)-f_{0}(980)$ mixing. In Ref. [10], we have updated the analysis of $\omega-\phi$ mixing in the framework of chiral perturbation theory with vector mesons. This result was used later in project C.2 in the investigation of isospin violation in the nucleon form factors [11]. As a basic ingredient for calculations in the meson-baryon sector, we have constructed the complete and minimal effective Lagrangian at one loop, the thirdorder terms are published in Ref. [12]. At third order, we find 78 independent operators, from which many, however, only contribute in processes with many pions and external fields. This minimal set of operators was later confirmed by the Murcia group [13]. We have also investigated the matching between the two- and three-flavor EFTs for the scalar sector [14]. Furthermore, we have calculated the hyperon vector form factors to one loop based on a covariant formulation of ChPT to study the possibility of extracting $V_{u s}$ [15]. We have also written a general review on "Chiral Perturbation Theory" for Annual Review in Nuclear and Particle Science [16].

\subsection{Kaon-nucleon scattering and hadronic atoms}

An important building block in the analysis of kaon photoand electroproduction is (anti)kaon-nucleon scattering. To further constrain the pertinent scattering amplitudes based on coupled-channel unitary extensions of ChPT, we have given the complete expression for the isospin-breaking corrections to the energy levels and the decay widths of kaonic hydrogen up-to-and-including $O\left(\alpha, m_{d}-m_{u}\right)$ in QCD+QED [17], with $\alpha$ the fine structure constant and $m_{q}$ the current mass of a quark of flavor $q$. It was demonstrated that although the leading-order corrections emerging due to the unitarity cusp are huge, they can be expressed solely in terms of the $\bar{K} N$ S-wave scattering lengths. We have also found additional large electromagnetic corrections and shown that the most recent DEAR data from kaonic hydrogen [18] are not consistent with most older scattering data (as it was also stressed by the Munich group). This extension of the leading-order Deser formula has become a standard tool in this field. These observations have further been sharpened in Ref. [19], where the strong $K^{-} p$ scattering length is extracted within chiral SU(3) unitary approaches from a very large variety of fits to low-energy $K^{-} p$ scattering data. For the first time, we have given theoretical uncertainties in such type of approach, based on a large number of Monte Carlo simulations in the multi-dimensional parameter-space (utilizing a method developed in project C.2). Within this scheme, a very good overall agreement with available scattering data is obtained (see Fig. 2) and the resulting scattering length is compared and found to be incompatible with the kaonic hydrogen data from DEAR at the $3 \sigma$ level. In Ref. [20], we have discussed the extraction of the S-wave kaonnucleon scattering lengths $a_{0}$ and $a_{1}$ from a combined analysis of existing kaonic hydrogen and synthetic deuterium data within the framework of a low-energy effective field theory. It turns out that with the present DEAR central values for the kaonic hydrogen ground-state energy and width, a solution for $a_{0}$ and $a_{1}$ exists only in a restricted domain of input values for the kaon-deuteron scattering length. It is thus very important to measure the kaon-deuteron scattering length, as proposed by the SIDDHARTA collaboration. Finally, we have shown how the nature of the $\Lambda(1520)$ as a dynamically generated resonance can be investigated in proton-proton collisions [21].

\subsection{Gauge-invariant formulation of coupled-channel dynamics}

Chiral unitary approaches have already been utilized successfully by various groups to describe kaon and $\eta$ photo- 

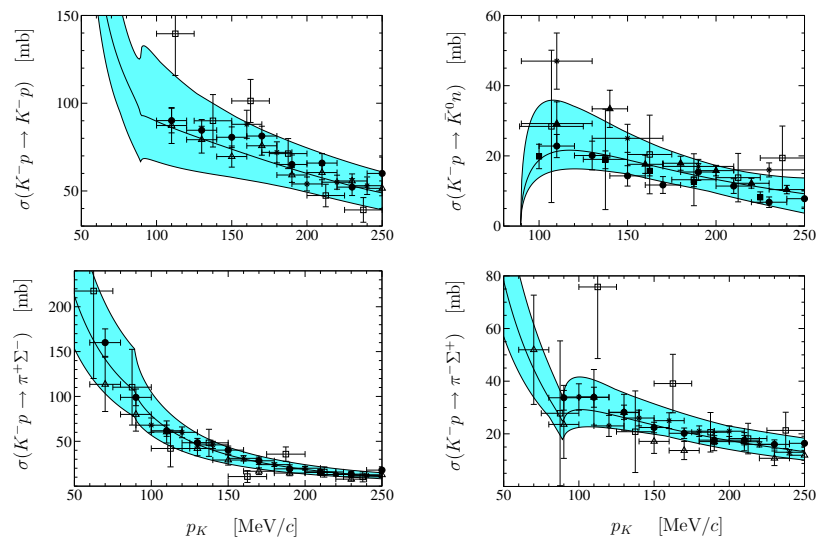

Figure 2. Total cross sections for $K^{-} p$ scattering into various channels. The best fit is represented by the solid line and the shaded area indicates the $1 \sigma$ confidence region. Figure taken from Ref. [19].

and electroproduction data off the nucleon. In most earlier calculations, the photon only scatters with the incoming baryon to form a meson-baryon pair that then undergoes strong final-state interactions. Such an approach is, however, not gauge invariant. In Ref. [22] we have shown how gauge invariance is obtained for the coupling of a photon to a two-body state described by the solution of the Bethe-Salpeter equation, i.e. the reaction $\gamma \phi B \rightarrow \phi B$ (with $\phi$ a Goldstone boson and $B$ a member of the ground state baryon octet). The key observation is that the photon has to couple to all possible initial, intermediate, and final states. The method has been illustrated both for a complex scalar field theory and for interaction kernels derived from chiral effective Lagrangians. This paves the way for a gauge-invariant formulation of meson photoproduction based on chiral coupled-channel dynamics. Subsequently, we have analyzed the so-called "turtle model" of electromagnetic meson production, which as the basic building block contains the full meson-baryon scattering T-matrix and the gauge-invariant subset of meson production vertices with an outgoing kaon. This model is gauge invariant (but not crossing symmetric). It is particularly useful to analyze the existing and upcoming (near threshold) kaon photo- and electroproduction data. As a first step, we have used it to test the various approximations (like the on-shell scheme for the loop integrals or the coupling of the photon to the initial baryon only) made in the literature so far [23].

\subsection{Phenomenological models of meson photoproduction and OZI analysis}

We have also performed some phenomenological studies of kaon and vector meson production based on results from SAPHIR (ELSA) and JLab. In Ref. [24] we studied the contribution of the Drell mechanism driven by $K^{+}$and $K^{-}$exchange to the reaction $\gamma N \rightarrow K \bar{K} N$. Adding a contribution from $K^{*}$-meson exchange with subsequent excitation of the $\Lambda(1520)$ resonance, we can describe the data of the older LAMP2 experiment. When applying the same model to the more recent SAPHIR data we find an excellent description of the $K^{+} p$ spectrum and evidence

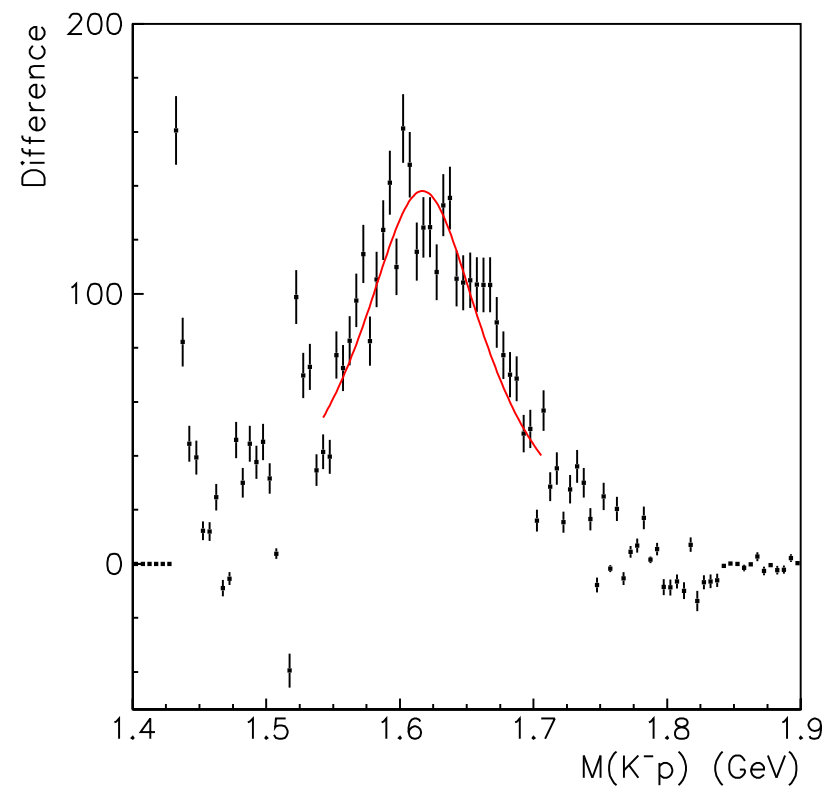

Figure 3. The difference between the SAPHIR data for the $K^{-} p$ invariant mass spectra of $\gamma p \rightarrow K^{+} K^{-} p$ and our model prediction. The solid line indicates the fit with a relativistic Breit-Wigner function. Figure taken from Ref. [24].

for a hyperon resonance with $M_{R}=(1617 \pm 2) \mathrm{MeV}$ and $\Gamma_{R}=(117 \pm 4) \mathrm{MeV}$ in the $K^{-} p$ mass distribution, see Fig. 3. We have also studied the violation of the OZI rule in photoproduction [25] and in proton-proton collisions [26]. Furthermore, we have given an explanation of the puzzling $A$-dependence in electromagnetic $\phi$-meson production off nuclei observed at Spring-8 based on a twostep mechanism [27].

\subsection{Coupled-channel dynamics in $\eta$ and $\eta^{\prime}$ decays}

Many of the technical aspects related to the work on (anti)kaon-nucleon scattering and photoproduction off nucleons can also be addressed in the decays of $\eta$ and $\eta^{\prime}$ mesons that exhibit strong final-state interactions. Besides, such reactions allow for fine tests of the symmetries of QCD. In Ref. [28] the hadronic decays $\eta, \eta^{\prime} \rightarrow 3 \pi$ and $\eta^{\prime} \rightarrow \eta \pi \pi$ were investigated within the framework of a U(3) chiral effective field theory in combination with a relativistic coupled-channel approach. Final-state interactions were included in the $\mathrm{S}$ - and $\mathrm{P}$-wave interactions. In Ref. [29] the claim that the light quark mass ratio $\left(m_{d}-m_{u}\right) / m_{s}$ can be extracted from the decay width ratio $\Gamma\left(\eta^{\prime} \rightarrow \pi^{0} \pi^{+} \pi^{-}\right) / \Gamma\left(\eta^{\prime} \rightarrow \eta \pi^{+} \pi^{-}\right)$was critically investigated. In particular, the assumption that the amplitude for $\eta^{\prime} \rightarrow \eta \pi \pi$ is constant over phase space is not correct, as shown by the Dalitz plot in the right panel of Fig. 4. Also, the assumption that the amplitude $A\left(\eta^{\prime} \rightarrow \pi^{0} \pi^{+} \pi^{-}\right)$is entirely given from the amplitude $A\left(\eta^{\prime} \rightarrow \eta \pi^{+} \pi^{-}\right)$through $\pi^{0}-\eta$ mixing could be shown to be erroneous. For further work on $\eta$ and $\eta^{\prime}$ decays in this framework, see Ref. [30]. 


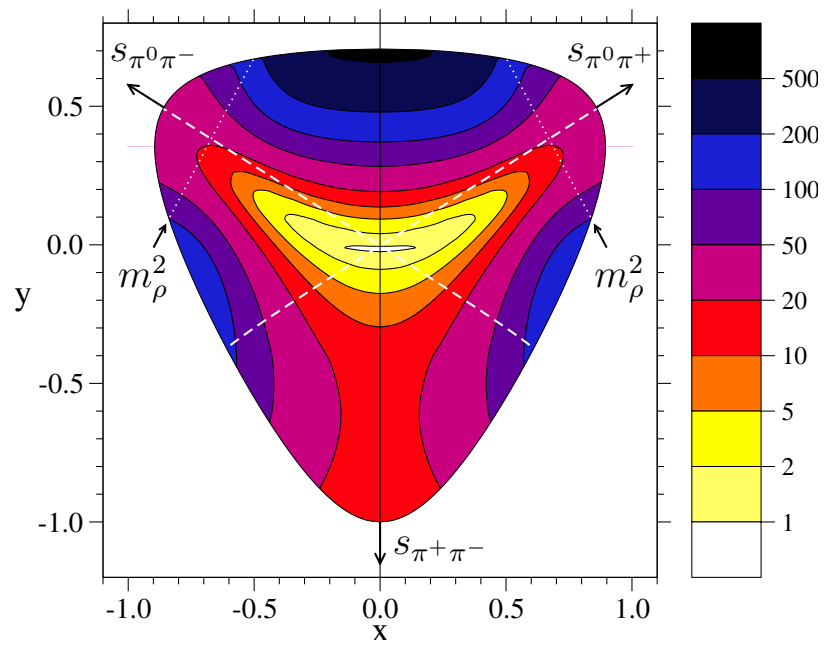

Figure 4. Dalitz plot distribution $\left|A\left(\eta^{\prime} \rightarrow \pi^{0} \pi^{+} \pi^{-}\right)\right|^{2}$ of the best overall fit including the VES data for $\eta^{\prime} \rightarrow \eta \pi^{+} \pi^{-}$. The distribution is normalized to unity at $x=y=0$. The P-wave contributions to $\pi^{0} \pi^{+}\left(\pi^{0} \pi^{-}\right)$rescattering vanish on the rising (falling) dashed line and the invariant energies associated with the $\rho^{ \pm}(770)$ are indicated by the dotted lines.

\subsection{Developments in chiral nuclear EFT and light nuclei}

To study also meson production off neutrons, it is mandatory to further develop the chiral EFT for few-nucleons systems. Furthermore, few-nucleon systems allow for fine tests of fundamental symmetries and their violation. In Ref. [31] the contributions from the isospin-violating one- and two-pion-exchange potentials to the nucleonnucleon potential were derived, in particular novel expressions for the subleading charge-symmetry-breaking twopion exchange potential were given. In Ref. [32] we have classified $A$-nucleon forces according to their isospin dependence and discussed the most general isospin structure of the three-nucleon force. We have derived the leading and subleading isospin-breaking corrections to the threenucleon force. Furthermore in Ref. [33] we have studied the two-nucleon force at next-to-next-to-leading order in a chiral EFT with explicit $\Delta$ degrees of freedom. Fixing the appearing LECs from a next-to-leading order calculation of pion-nucleon threshold parameters, we find an improved convergence of most peripheral nucleon-nucleon phases compared to the theory with pions and nucleons only. We have also studied the consistency of Weinberg's power counting [34] and further elaborated on the conjectured infrared renormalization group limit cycle in QCD $[35,36]$. To extend this program to nuclei beyond $A \geq 5$, we have started a long-term project in nuclear lattice simulations, which amounts to a lattice formulation of the $A$-nucleon Schrödinger equation and its solution via Monte Carlo methods, utilizing the potentials derived in chiral EFT. First results obtained for the $A=2,3,4$ systems already looked promising [37, 38]. Finally, universal properties of few-body systems have been studied in Refs. [39, 40]. In particular, in the investigation of the triton charge form factor [39], the nucleon form factors developed in project $\mathbf{C} .2$ were used. In a similar fashion, the

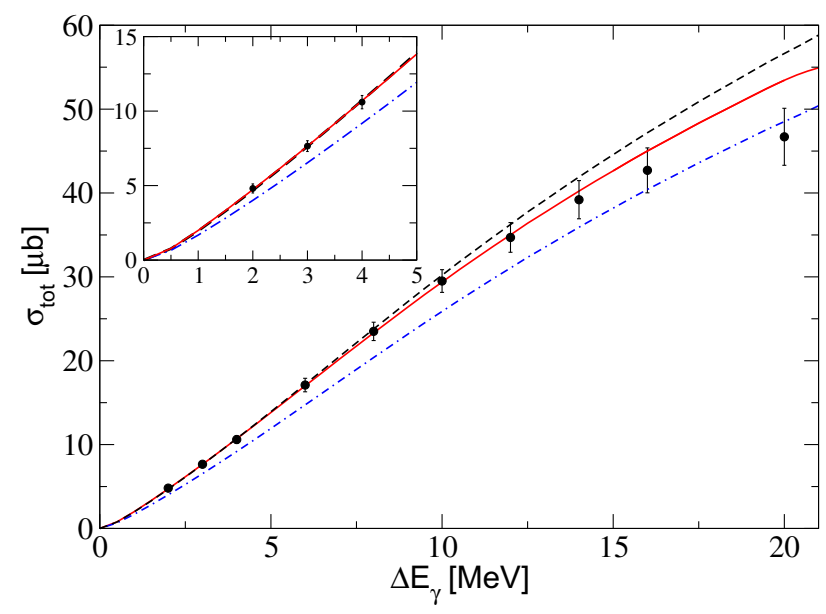

Figure 5. Total cross section of the reaction $\gamma d \rightarrow \pi^{+} n n$ at LO (dashed line), NLO (dash-dotted line) and NNLO (solid line) together with experimental data from MIT-Bates. Figure taken from Ref. [42].

isospin mixing effects in the nucleon form factors worked out in project $\mathbf{C} .2$ were used to analyze isospin mixing in ${ }^{4} \mathrm{He}$ and its influence on the extraction of the nucleon strange electric form factors [41].

\subsection{Pion photoproduction off deuterium}

In Ref. [42] we have analyzed the reaction $\gamma d \rightarrow \pi^{+} n n$ up to order $\chi^{5 / 2}$ in ChPT, where $\chi$ denotes the ratio of the pion to the nucleon mass. Special emphasis was put on the role of nucleon-recoil corrections that are the source of contributions with fractional power in $\chi$. Using the threshold production amplitude for $\gamma p \rightarrow \pi^{+} n$ calculated in ChPT a long time ago by Bernard, Kaiser, and Meißner as the only input, the total cross section for $\gamma d \rightarrow \pi^{+} n n$ is described very well, see Fig. 5. A conservative estimate suggests that the theoretical uncertainty for the transition operator amounts to $3 \%$ for the computed amplitude near threshold. In Ref. [43] we have discussed the extraction of the neutron-neutron scattering length from experimental spectra on this reaction. We show that for properly chosen kinematics, the theoretical uncertainty of the method can be as low as $0.1 \mathrm{fm}$.

\section{Second funding period 2008-2012}

\subsection{Chiral symmetry tests in Goldstone boson decays}

One of the highlights of the previous funding period were new developments for precision determinations of the pion-pion scattering lengths. One method exploits the unitarity cusp at the $\pi^{+} \pi^{-}$threshold in the $\pi^{0} \pi^{0}$ spectrum in the decay $K^{+} \rightarrow \pi^{0} \pi^{0} \pi^{+}$, which has been measured with unprecedented precision by the NA48/2 collaboration at CERN; see Fig. 6. To interpret this cusp effect in a model-independent manner and extract the scattering length combination $a_{0}^{0}-a_{0}^{2}$ with highest possible precision, we have developed a manifestly covariant version of 


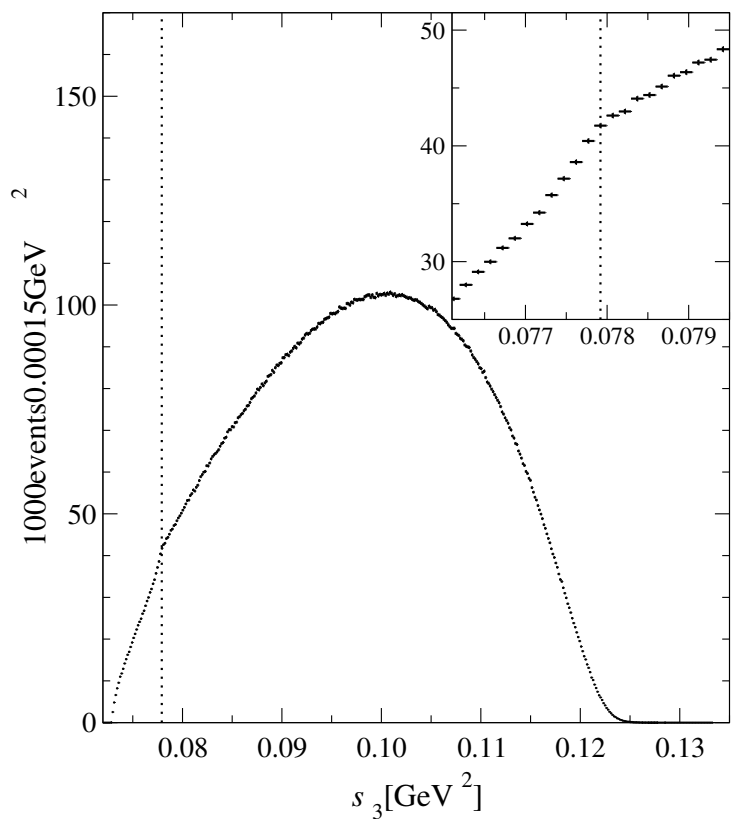

Figure 6. Cusp in the decay spectrum $d \Gamma / d s_{3}$ of the decay $K^{ \pm} \rightarrow$ $\pi^{0} \pi^{0} \pi^{ \pm}$as seen by the NA48/2 collaboration. The dotted vertical line marks the position of the $\pi^{+} \pi^{-}$threshold, the insert focuses on the cusp region. Figure taken from Ref. [44], based on data from Ref. [45].

non-relativistic effective field theory (NREFT) $[6,46]$, extending and partly relying on the expertise on NREFTs in the context of hadronic atoms [47]. An essential ingredient of the most recent experimental analysis [48] was the inclusion of radiative corrections [49], which have an important impact on the analytic structure of the decay amplitude near the cusp. The group's expertise on radiative corrections was subsequently also used for a different class of kaon decays, the flavor-changing neutral current processes $K \rightarrow \pi \ell^{+} \ell^{-}$[50]. The theoretical foundations of this theory were recently clarified [51], including thorough investigations of the analytic properties of the decay amplitude at two-loop order. Furthermore, a similar cusp effect has also been predicted and analyzed for the decay $\eta^{\prime} \rightarrow \eta \pi^{0} \pi^{0}[52]$.

A complementary determination of $\pi \pi$ scattering lengths is based on $K_{e 4}$ decays. It has been pointed out that also here, isospin-breaking corrections play an important role [53], given the tremendous precision of the data collected at NA48/2 [54]. The combination of both kaondecay-based scattering length extractions has led to the most precise experimental determinations of these fundamental quantities in low-energy strong-interaction physics to date, in excellent agreement with theoretical predictions [55].

Chiral perturbation theory, as a perturbative expansion of observables in the light quark masses, in particular allows to study the pattern of explicit chiral symmetry breaking caused thereby, giving unique access to the masses of the up, down, and strange quarks, which are not otherwise accessible due to their confinement inside hadrons. A particularly important process to constrain the ratios of the light quark masses is the isospin-breaking decay $\eta \rightarrow 3 \pi$.

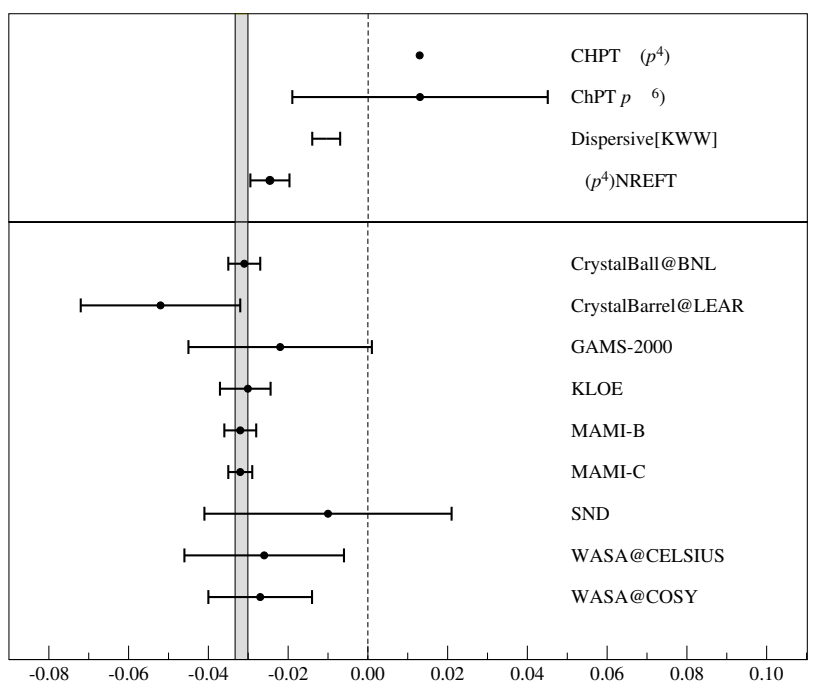

Figure 7. Comparison of values for the slope parameter $\alpha$ in $\eta \rightarrow 3 \pi^{0}$. Top: theoretical predictions. Bottom: experimental determinations. The gray shaded area is the average from Ref. [63]. Figure taken from [62].

One of its particular advantages is the suppression of electromagnetic (as opposed to $m_{u}-m_{d}$ ) effects; these have been reassessed to higher precision than before [56], but still finding only very small corrections. In order to extract the quark mass ratio from the normalization of the measured $\eta \rightarrow 3 \pi$ Dalitz plot distribution, the energy dependence of the decay amplitude has to be under control with very high precision-a notoriously difficult task for chiral perturbation theory in particular due to the large pion-pion rescattering effects. One particular issue in the past few years was a discrepancy between the $\eta \rightarrow 3 \pi^{0}$ Dalitz plot parameter $\alpha$ as measured with very high precision by various experiments (analyzed in the context of the TR16 project C.5 [57] and elsewhere [58-60]), and as predicted by chiral perturbation theory [61]. We have made use of the NREFT developed for $K \rightarrow 3 \pi$ decays to study these corrections [62], giving a theoretical prediction for $\alpha$ compatible with experimental findings, and explaining the failure of chiral perturbation theory for this observable at the same time; see Fig. 7 for an illustration. Also, we have derived a model-independent relation between the Dalitz plot parameters for the charged- and neutral-pion final state that seems to be violated by the most recent (and accurate) $\eta \rightarrow \pi^{+} \pi^{-} \pi^{0}$ data [64].

Also in connection with the ongoing $\eta / \eta^{\prime}$ program in project $\mathbf{C . 5}$ and at various other laboratories, we have predicted the branching ratios of the yet unmeasured anomalous decays of $\eta^{\prime}$ and $\eta$ into four pions [65]. For the Pwave-dominated final states including charged pions, we predicted branching fractions only slightly below the upper limits at the time; first experimental measurements of these by the BESIII Collaboration found agreement with our predictions within uncertainties [66]. On the other hand, the decays into neutral pions only have to be $\mathrm{D}$-wave driven as long as CP symmetry is conserved, and are therefore more strongly suppressed. CP-violating contributions to these decays based on the QCD $\theta$-term have also been 
considered (the bounds on which were recently re-studied in the context of the neutron electric dipole moment [67]).

When going to higher energies, the purely perturbative treatment of final-state interactions in meson decays, be it in chiral perturbation theory or the NREFT framework, are doomed to fail, and one has to resort to methods from dispersion theory that resum such rescattering effects to all orders. We have performed such a dispersive analysis for the three-pion decays of the lightest isoscalar vector mesons $\omega$ and $\phi$ [68], allowing for a consistent description of final-state interactions between all three pions. We calculated the Dalitz plot distributions for both decays, comparing to the high-precision experimental determinations of $\phi \rightarrow 3 \pi$ by KLOE [69] and CMD-2 [70], and predicting the $\omega \rightarrow 3 \pi$ Dalitz plot about to be analyzed at WASA-atCOSY, KLOE, CLAS, or elsewhere. As a further application of this study, we have used the resulting three-pion decay amplitudes, together with the vector form factor of the pion, as input to predict the vector-meson conversion decays $\omega \rightarrow \pi^{0} \ell^{+} \ell^{-}$and $\phi \rightarrow \pi^{0} \ell^{+} \ell^{-}$[71].

Dispersive methods have also been employed to demonstrate a new, model-independent way to analyze the decays $\eta, \eta^{\prime} \rightarrow \pi^{+} \pi^{-} \gamma$ [72], which are also driven by the chiral anomaly. This has been applied to experimental data by the WASA-at-COSY [73] and subsequently by the KLOE [74] collaborations.

\subsection{Chiral coupled-channel analysis of meson-baryon scattering and meson production}

Working in a still perturbative setting, we have analyzed meson-baryon scattering lengths in the framework of covariant $\mathrm{SU}(3)$ baryon chiral perturbation theory at leading one-loop order [75]. We have computed the complete set of matching relations between the dimension-two lowenergy constants in the two- and three-flavor formulations of the theory, and derived new two-flavor low-energy theorems for pion-hyperon and pion-cascade scattering that have already been tested in lattice simulations [76].

As the convergence behavior of three-flavor chiral perturbation theory in the baryon sector is doubtful at best already at threshold, unitarization methods can be used to extend chiral amplitudes to higher energies and investigate properties of resonances [77-79]. This nicely connects to the experimental and partial-wave-analysis resonance studies performed in the projects A.1, A.2, and B.1. We remark, however, that a systematic investigation of such resonance generation has to start by considering the lowlying resonances. In the last funding period, we have made considerable progress within this demanding field:

$S_{11}$ resonances [77]: We have analyzed S-wave pionnucleon scattering in a unitarized chiral effective Lagrangian including all dimension-two contact terms. We find that both the $S_{11}(1535)$ and the $S_{11}(1650)$ are dynamically generated, see Fig. 8, but the $S_{31}(1620)$ is not. This is a novel result. We have also analyzed the structure of these dynamically generated resonances in terms of their meson-baryon components.

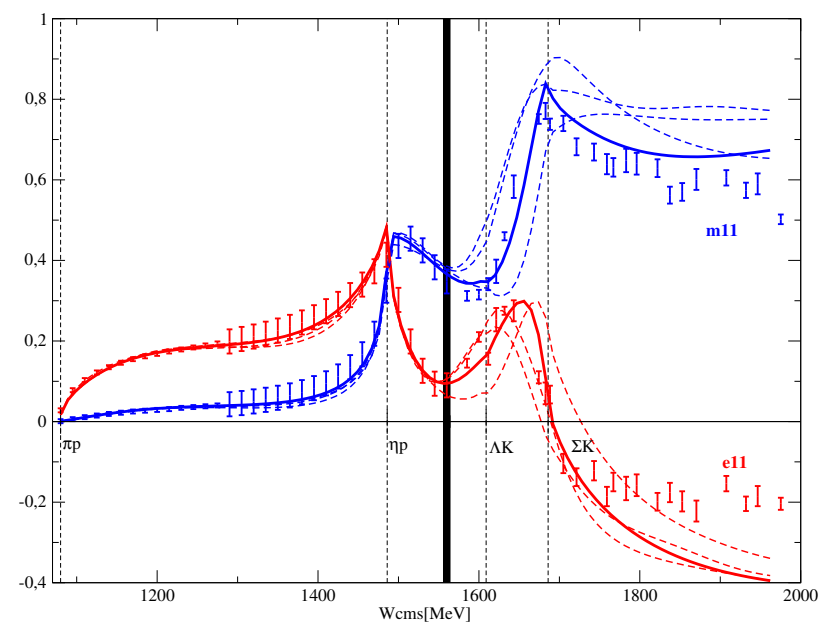

Figure 8. Real and imaginary part of the $S_{11}$ partial wave amplitude compared with the SAID-data (WI08-analysis). Full curves correspond to the best fit, the dashed ones to fits with slightly worse $\chi_{\text {dof }}^{2}$. The bold vertical line limits the region of the fit, where in the non-fit region single energy values are taken from the SAID-data. Figure taken from Ref. [77].

$\eta$ photoproduction [78]: We have analyzed photoproduction of $\eta$ mesons off the proton in a gauge-invariant chiral unitary framework. The interaction kernel for mesonbaryon scattering was derived from the leading order chiral effective Lagrangian and iterated in a Bethe-Salpeter equation. The recent precise threshold data from Crystal Ball at MAMI [80] can be described rather well, and the complex pole corresponding to the $S_{11}(1535)$ was extracted. An extension of the kernel to include magnetic couplings was also discussed.

Structure of the $\Lambda(1405)$ [79]: We have performed a combined analysis of antikaon-nucleon scattering cross sections and the recent SIDDHARTA kaonic hydrogen data [81] in the framework of a coupled-channel BetheSalpeter approach at next-to-leading order in the chiral expansion of the effective potential. We find a precise description of the antikaon-proton scattering amplitudes and were able to extract accurate values of the scattering lengths, $a_{0}=-1.81_{-0.28}^{+0.30}+i 0.92_{-0.23}^{+0.29} \mathrm{fm}, a_{1}=+0.48_{-0.11}^{+0.12}+$ $i 0.87_{-0.20}^{+0.26} \mathrm{fm}$. We have also shed new light on the two-pole structure of the $\Lambda(1405)$.

Polarization observables in $\gamma p \rightarrow \pi^{0} \eta p$ [82]: We evaluate the polarization observables $I^{S}$ and $I^{C}$ for the reaction $\gamma p \rightarrow \pi^{0} \eta p$, using a chiral unitary framework. These observables have been recently measured for the first time by the CBELSA/TAPS collaboration (see project A.1). The theoretical predictions of $I^{S}$ and $I^{C}$, given for altogether 18 angle dependent functions, are in good agreement with the measurements; see Fig. 9. Also, the asymmetry $d \Sigma / d \cos (\theta)$ agrees with the data. We show the importance of the $\Delta(1700) D_{33}$ resonance and its S-wave decay into $\eta \Delta(1232)$. The result can be considered as a further confirmation of the dynamical nature of this resonance. At the highest energies, deviations of the predictions from the data start to become noticeable, which leaves room for additional processes and resonances such as a $\Delta(1940) D_{33}$. 


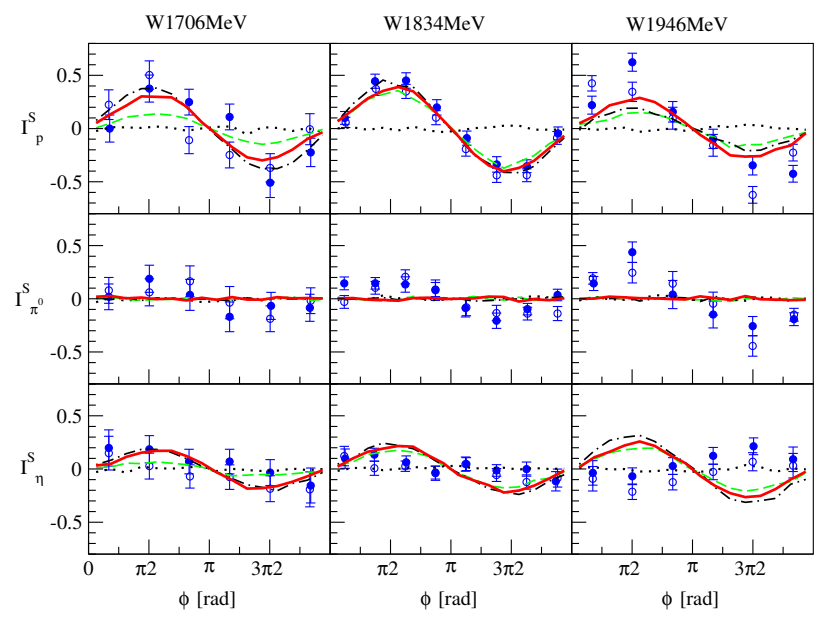

Figure 9. Polarization observable $I^{S}\left(\phi^{*}\right)$ for the three cases of $p, \pi^{0}$, and $\eta$ spectators and for three different energies $W \equiv \sqrt{s}$. The data $I^{S}\left(\phi^{*}\right)$ are from Ref. [83] (full circles). The empty circles show $-I^{S}\left(2 \pi-\phi^{*}\right)$. (Red) solid lines: Present results, predicted from the model of Refs. [84, 85]. (Black) dotted lines: Without the $\Delta(1700) \eta \Delta$ and $\Delta(1700) K \Sigma(1385)$ couplings predicted from the chiral unitary model. (Green) dashed lines: Without the contributions from the $\pi^{0} S_{11}(\eta p)$ final state. (Black) dash-dotted lines: Only contribution from the $\Delta(1700) \eta \Delta$ and $\Delta(1700) K \Sigma(1385)$ tree graphs. Figure taken from Ref. [82].

\section{Third funding period 2012-2016}

\subsection{Precision studies in light meson decays}

In the realm of light-meson physics, most of the work performed in the final funding period has been motivated by hadronic contributions to the anomalous magnetic moment of the muon. In particular, the hadronic virtual corrections denoted by "hadronic light-by-light scattering" (HLbL) have so far only been investigated in model calculations. In view of the upcoming $(g-2)_{\mu}$ experiments at Fermilab and JPARC, which are expected to improve on the accuracy achieved by the BNL E821 experiment by roughly a factor of four, an improved determination of HLbL contributions with a reliable assessment of the uncertainty is urgently called for. We have therefore suggested to conduct an analysis of at least the lightest, dominant intermediate states using dispersion theory [86-88], thereby reducing the problem to a data-driven driven analysis, much in the same spirit as the relation of hadronic vacuum polarization to $e^{+} e^{-} \rightarrow$ hadrons cross section measurements. We have therefore investigated several photo-pion and $\eta, \eta^{\prime}$ processes that are important in this respect.

For the reaction $\gamma \gamma \rightarrow \pi \pi$, Roy-Steiner equations (see the more detailed discussion in the context of pionnucleon scattering in Sec. 4.4) have been worked out and solved numerically [89]. The subtraction constants introduced to reduce the influence of high-energy input can be identified with the pion dipole and quadrupole polarizabilities. A sum rule for the isospin $I=2 \mathrm{~S}$-wave, together with chiral constraints, produces an improved prediction for the charged-pion quadrupole polarizability $\left(\alpha_{2}-\beta_{2}\right)^{\pi^{ \pm}}=(15.3 \pm 3.7) \cdot 10^{-4} \mathrm{fm}^{5}$. The two-photon coupling of the $\sigma$ resonance was investigated and a correlation

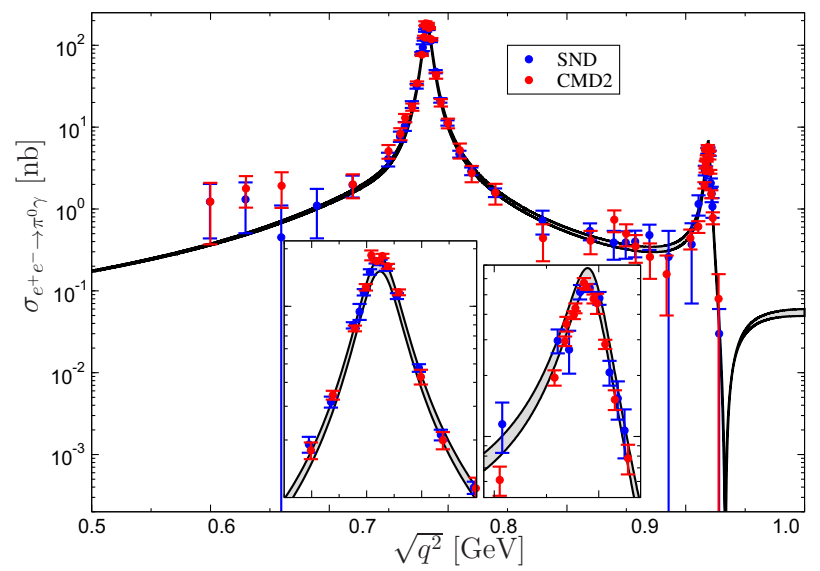

Figure 10. $e^{+} e^{-} \rightarrow \pi^{0} \gamma$ cross section predicted from $e^{+} e^{-} \rightarrow 3 \pi$, compared to data [92-94]. The inserts zoom in on the $\omega$ and $\phi$ resonance peaks. Figure taken from Ref. [91].

with the isospin $I=0$ pion polarizabilities derived, which, using the chiral-perturbation-theory results for the polarizabilities, led to $\Gamma_{\sigma \gamma \gamma}=(1.7 \pm 0.4) \mathrm{keV}$.

The process $\gamma \pi \rightarrow \pi \pi$ is driven by the chiral anomaly, and hence a consequence of one of the most profound predictions of QCD at low energies. In contrast to the decay $\pi^{0} \rightarrow \gamma \gamma$, similarly anomalous, that has been well tested experimentally, the prediction for $\gamma \pi \rightarrow \pi \pi$ has never been verified better than to a level of about $10 \%$. We have therefore suggested a dispersive representation that will allow a much more accurate experimental extraction of the chiral anomaly from Primakoff data as taken by the COMPASS collaboration, as it allows to use not only the (scarce) nearthreshold data, but at least the full range in which pionpion scattering is still elastic to excellent approximation, hence ranging up to energies of about $1 \mathrm{GeV}$, and therefore including the resonant enhancement by the $\rho$ [90].

Subsequently, we have performed the first dispersive analysis of the $\pi^{0}$ transition form factor [91], with so far evaluating the singly-virtual one for both timelike and spacelike momenta. For isovector intermediate states, the previously discussed amplitude for $\gamma \pi \rightarrow \pi \pi$ serves as a vital input. For the isoscalar part, we have generalized the study of $\omega / \phi \rightarrow 3 \pi[68]$ to arbitrary virtualities in $e^{+} e^{-} \rightarrow 3 \pi$, fitting the available cross section data. The resulting prediction for $e^{+} e^{-} \rightarrow \pi^{0} \gamma$ yields excellent agreement with direct measurements, see Fig. 10. The prediction at (moderate) spacelike momenta will soon be tested by new data from the BESIII collaboration. We can also predict the slope of the singly-virtual transition form factor at vanishing virtuality with unprecedented accuracy, using a sum rule, with the result $a_{\pi}=(30.7 \pm 0.6) \times 10^{-3}$.

Vector meson conversion decays, such as $\omega \rightarrow \pi^{0} \ell^{+} \ell^{-}$ or $\phi \rightarrow \eta \ell^{+} \ell^{-}$, pose one of the few opportunities to test the doubly-virtual transition form factors (of $\pi^{0}$ and $\eta$, respectively, in the examples given) experimentally with good precision. In particular data on $\omega \rightarrow \pi^{0} \mu^{+} \mu^{-}$for large dimuon invariant masses seems to be in sharp contrast with all theory predictions, as already noted in our own dispersive analysis [71]. As the latter relies on elastic unitarity, we have also investigated general "unitarity 
bounds" on this form factor, confirming the existence of a disagreement with experimental data near the upper end of the decay region [95]. This disagreement can be further sharpened when using cross section data on $e^{+} e^{-} \rightarrow \omega \pi^{0}$ explicitly [96].

The previous dispersive analysis of the $\eta$ (and $\eta^{\prime}$ ) singly-virtual transition form factor by the Jülich group [97] builds on the model-independent representation of the decay amplitude $\eta \rightarrow \pi \pi \gamma$ [72]. We have improved the latter representation by the inclusion of the leading left-hand-cut contribution, the $a_{2}$ (1320) tensor resonance (that decays into $\pi \eta$ ) [98], and shown that some highly interesting aspects of this representation could be tested in a very useful manner by a measurement of the crossedchannel reaction $\gamma \pi \rightarrow \pi \eta$-an experiment that is feasible, in a Primakoff reaction, at the COMPASS experiment at CERN. Finally, first steps towards a test of the doublyvirtual $\eta$ transition form factor have been taken, testing the factorization hypothesis in data on $e^{+} e^{-} \rightarrow \eta \pi \pi$ [99]. A central result is that the left-hand-cut contributions calculated in Ref. [98] are a vital input to allow for (approximate) factorization, which would have to be broken badly without it.

\subsection{Antikaon-nucleon scattering revisited}

We have re-analyzed meson-baryon scattering amplitude in the strangeness $S=-1$ sector in Ref. [100] because of the new SIDDHARTA data and similar NLO work by other groups [101, 102]. The free parameters of our approach, the low-energy constants as well as the regularization scales $\mu$ are adjusted to reproduce all known experimental data in the meson-baryon sector. The main bulk of this data consists of the cross sections for the processes $K^{-} p \rightarrow K^{-} p, K^{-} p \rightarrow \bar{K}^{0} n, K^{-} p \rightarrow \pi^{0} \Lambda$, $K^{-} p \rightarrow \pi^{+} \Sigma^{-}, K^{-} p \rightarrow \pi^{0} \Sigma^{0}$, and $K^{-} p \rightarrow \pi^{-} \Sigma^{+}$for laboratory momentum $P_{\text {lab }}<300 \mathrm{MeV}$. Electromagnetic effects are not included in the analysis and assumed to be negligible at the measured values of $P_{\text {lab. Addition- }}$ ally, at the antikaon-nucleon threshold, the decay ratios $\Gamma\left(K^{-} p \rightarrow \pi^{+} \Sigma^{-}\right) / \Gamma\left(K^{-} p \rightarrow \pi^{-} \Sigma^{+}\right)=2.38 \pm 0.04$, $\Gamma\left(K^{-} p \rightarrow \pi^{0} \Lambda\right) / \Gamma\left(K^{-} p \rightarrow\right.$ neutral $)=0.189 \pm 0.015$, and $\Gamma\left(K^{-} p \rightarrow \pi^{ \pm} \Sigma^{\mp}\right) / \Gamma\left(K^{-} p \rightarrow\right.$ inelastic $)=0.664 \pm 0.011$, as well as the energy shift and width of kaonic hydrogen in the 1 s state, i.e. $\Delta E-i \Gamma / 2=(283 \pm 42)-i(271 \pm 55) \mathrm{eV}$ from the SIDDHARTA experiment at DAФNE, were considered. The fit procedure was performed in two steps: First, for randomly chosen starting values of the free parameters (in a natural range) the fit was performed to all threshold values and the cross section data at a few momenta $P_{\text {lab }}<300 \mathrm{MeV}$. Repeating this procedure several thousand times, we ended with several dozen of parameter sets that describe the data equally well. For each of these sets the amplitudes were analytically continued to the positive and negative complex plane. Thereafter, every unphysical solution, e.g. poles on the first Riemann sheet for $\operatorname{Im}(W)<200 \mathrm{MeV}$, was sorted out. The remaining sets were used in the second step as starting point of the fit procedure, including all threshold and cross section data

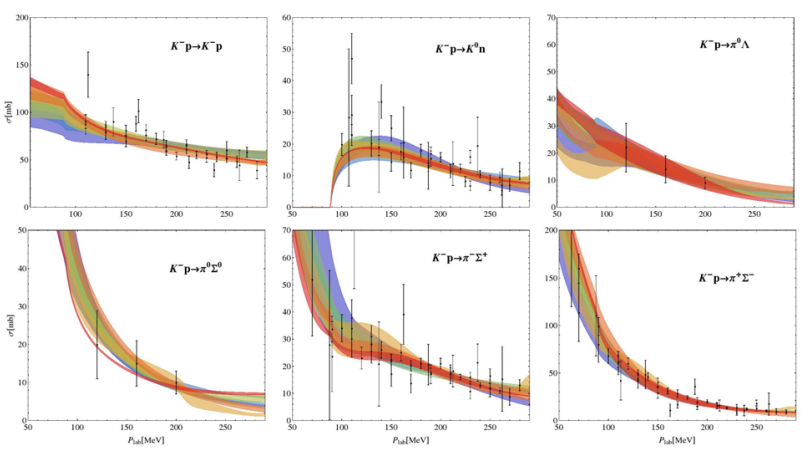

Figure 11. Fit results compared to the experimental data of $K^{-}$proton scattering. Different colors correspond to the eight best solutions, while the bands represent the $1 \sigma$ uncertainty due to errors of the fit parameters. Figure taken from Ref. [100].

points. Eight best solutions were obtained by this procedure, see Table 1, whereas the next best $\chi_{\text {d.o.f. }}^{2}$ are at least one order of magnitude larger. Although the fit results look very promising, see Fig. 11, we would like to point out that there are quite a few free parameters in the model. The latter are assumed to be of natural size, but not restricted otherwise. When continued analytically to the complex $W$ plane, all eight solutions confirm the double pole structure of the $\Lambda(1405)$. Due to the SIDDHARTA data restricting the amplitude close to the the $\bar{K} N$ threshold quite strongly, we observe stability of the position of the narrow pole. The position of the second pole is, on the other hand, less restricted. Therefore, further data are needed to resolve this ambiguity.

We have further presented a comparative analysis of the various chiral unitary SU(3) approaches to (anti)kaonnucleon scattering [103]. It was demonstrated that the approaches lead to very different predictions for the $K^{-} p$ amplitude extrapolated to subthreshold energies as well as for the $K^{-} n$ amplitude. The origin of the poles generated by the models is traced to the so-called zero-coupling limit, in which the inter-channel couplings are switched off. This provides new insights into the pole contents of the various approaches.

\subsection{The $\Lambda(1405)$ in kaon photoproduction}

Recently, very sophisticated measurements of the reaction $\gamma p \rightarrow K^{+} \Sigma \pi$ were performed by the CLAS collaboration at JLAB [104]. There, the invariant mass distribution of all three $\pi \Sigma$ channels was determined in a broad energy range and with high resolution. Finally, from these data the spin-parity analysis of the $\Lambda(1405)$ was performed in Ref. [105]. There, for the first time, the quantum numbers $J^{P}=1 / 2^{-}$were deduced from an experimental measurement directly. As a coupled-channel gauge invariant chiral unitary model is very complicated, we have assumed the simplest ansatz for the photoproduction amplitude to analyze the CLAS data [100]

$$
\mathcal{M}^{j}\left(\tilde{W}, M_{\mathrm{inv}}\right)=\sum_{i=1}^{10} C^{i}(\tilde{W}) G^{i}\left(M_{\mathrm{inv}}\right) f_{0+}^{i, j}\left(M_{\mathrm{inv}}\right),
$$


Table 1. Quality of the various fits in the description of the hadronic and the photoproduction data from CLAS. For the precise definition of $\chi_{\text {p.p. }}^{2}$, see Ref. [100].

\begin{tabular}{|c|cccccccc|}
\hline Fit \# & 1 & 2 & 3 & 4 & 5 & 6 & 7 & 8 \\
\hline$\chi_{\text {d.o.f. }}^{2}($ hadronic data $)$ & 1.35 & 1.14 & 0.99 & 0.96 & 1.06 & 1.02 & 1.15 & 0.90 \\
$\chi_{\text {p.p. }}^{2}($ CLAS data $)$ & 3.18 & 1.94 & 2.56 & 1.77 & 1.90 & 6.11 & 2.93 & 3.14 \\
\hline
\end{tabular}

where $\tilde{W}$ and $M_{\text {inv }}$ denote the total energy of the system and the invariant mass of the $\pi \Sigma$ subsystem, respectively. For a specific meson-baryon channel $i$, the energy-dependent (and in general complex valued) constants $C^{i}(\tilde{W})$ describe the reaction mechanism of $\gamma p \rightarrow$ $K^{+} M_{i} B_{i}$, whereas the final-state interaction is captured by the standard Höhler partial waves $f_{0+}$. For a specific meson-baryon channel $i$, the Green's function is denoted by $G^{i}\left(M_{\text {inv }}\right)$. The only new parameters of the photoproduction amplitude are the constants $C^{i}(\tilde{W})$ which, however, are quite numerous (10 for each $\tilde{W})$. These parameters are adjusted to reproduce the invariant mass distribution $d \sigma / d M_{\text {inv }}\left(M_{\text {inv }}\right)$ for the final $\pi^{+} \Sigma^{-}, \pi^{0} \Sigma^{0}$, and $\pi^{-} \Sigma^{+}$states and for all 9 measured total energy values $\tilde{W}=2.0, \ldots, 2.8 \mathrm{GeV}$. The achieved quality of the photoproduction fits is listed in the third row of Table 1, where the $\chi_{\text {d.o.f. }}^{2}$ of the hadronic part are stated in the second row. Note that for the comparison of the photoproduction fits the quantity $\chi_{\text {d.o.f. }}^{2}$ is not meaningful due to the large number of generic parameters $C_{i}(\tilde{W})$. Therefore, we compare the total $\chi^{2}$ divided by the total number of data points for all three $\pi \Sigma$ final states, denoted by $\chi_{\text {p.p. }}^{2}$. It turns out that even within such a simple and flexible photoproduction amplitude, only the solutions \#2, \#4, and \#5 of the eight hadronic solutions allow for a decent description of the CLAS data. The best solution is indeed \#4, which we display in Fig. 12. Incidentally, it also has the lowest $\chi_{\text {d.o.f. }}^{2}$ for the hadronic part. This solution also gives an excellent description of the $\Sigma \pi \pi$ mass distribution from Ref. [106]. With respect to these data, solution \#2 is also satisfactory but \#5 is not. Therefore, the photoproduction data combined with the scattering and the SIDDHARTA data lead to a sizable reduction in the ambiguity of the second pole of the $\Lambda(1405)$. To be precise, the location of the two poles in these surviving solutions is given by (all energies in $\mathrm{MeV}$ )

sol. \#2, pole $1: 1434_{-2}^{+2}-i 10_{-1}^{+2}$, pole $2: 1330_{-5}^{+4}-i 56_{-11}^{+17}$,

sol. \#4, pole $1: 1429_{-7}^{+8}-i 12_{-3}^{+2}$, pole $2: 1325_{-15}^{+15}-i 90_{-18}^{+12}$.

We conclude that the inclusion of the CLAS data as experimental input can serve as a new important constraint on the antikaon-nucleon scattering amplitude. However, for future studies a theoretically more robust model for the two-meson photoproduction amplitude is required. Finally, note that the two-pole structure of the $\Lambda(1405)$ is now also discussed in the Particle Data Group [107].
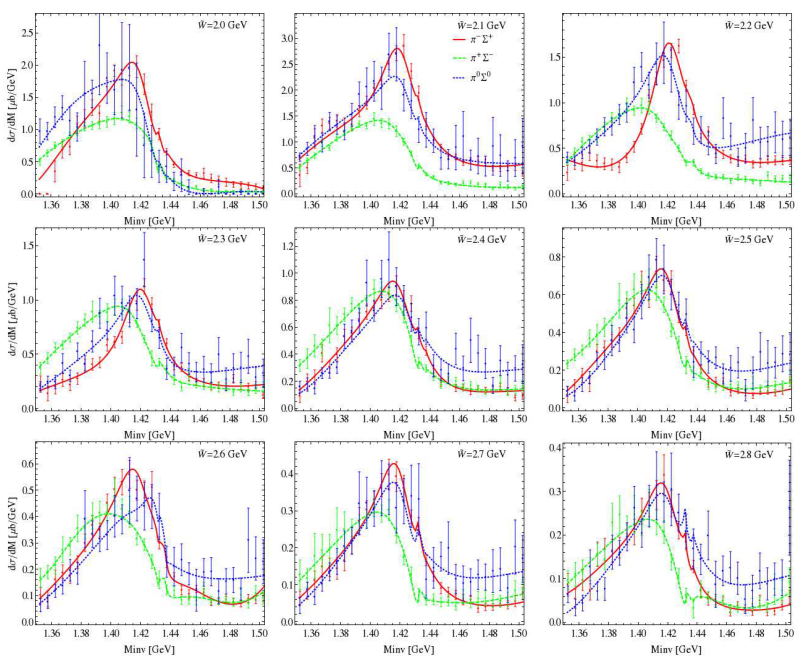

Figure 12. Result of the fits to the CLAS data in all three channels $\pi^{+} \Sigma^{-}$(green), $\pi^{-} \Sigma^{+}$(red) and $\pi^{0} \Sigma^{0}$ (blue). Correspondingly, green (dashed), red (full) and blue (dotted) lines represent the outcome of the model for the solution \#4 in the $\pi^{+} \Sigma^{-}, \pi^{-} \Sigma^{+} \pi^{0} \Sigma^{0}$ channels, respectively. Figure taken from Ref. [100].

\subsection{Pion-nucleon scattering: from scattering lengths to Roy-Steiner equations}

In a series of publications [108-111], we have made substantial progress in understanding and determining the (near-)threshold properties of pion-nucleon scattering. In a first step, the complete isospin-breaking corrections, both induced by the light quark-mass difference and electromagnetic effects, were calculated for the pion-nucleon scattering lengths in all physical channels, using covariant baryon chiral perturbation theory [108]. These results were then extended slightly above threshold, so that the energy dependence of the so-called triangle relation (between the three physical channels accessible in chargedpion-proton scattering) could be investigated [109]. Subsequently, we have calculated $\pi^{-}$deuteron scattering at threshold to percent-level accuracy, including isospinviolating corrections both in the two- and three-body sector $[110,111]$. This was used to extract the isoscalar and isovector pion-nucleon scattering lengths from data on pionic hydrogen [112] and pionic deuterium [113] atoms, leading to the results $a^{+}=(7.6 \pm 3.1) \times 10^{-3} M_{\pi}^{-1}$ and $a^{-}=(86.1 \pm 0.9) \times 10^{-3} M_{\pi}^{-1}$, thus indicating a nonvanishing (and positive) $a^{+}$at $2 \sigma$ significance; see Fig. 13 . Finally, we have discussed the validity of the GoldbergerMiyazawa-Oehme sum rule in the presence of isospin vio- 


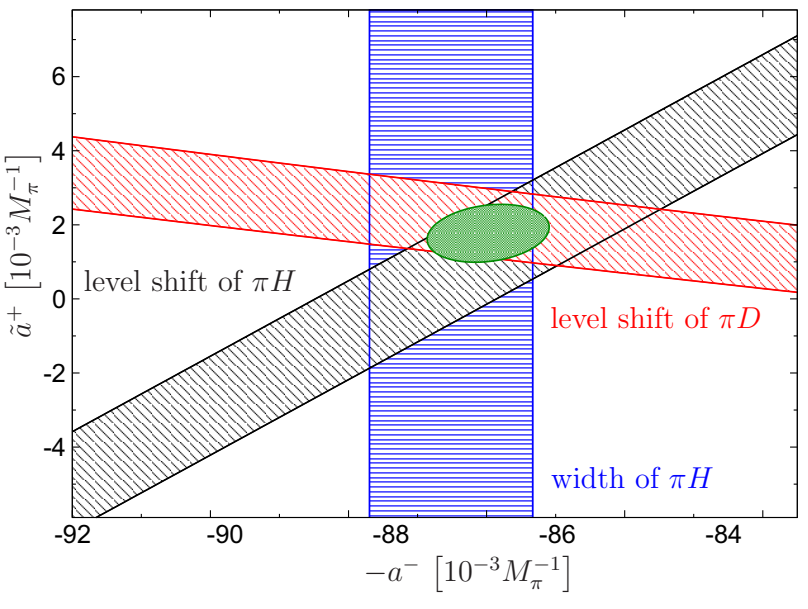

Figure 13. Combined constraints on $\tilde{a}^{+}$and $a^{-}$from data on the width and level shift of $\pi H$, as well as the $\pi D$ level shift. The figure is an updated version of the ones from Refs. [110, 111], taken from Ref. [114], to account for the new value of the $\pi H$ level shift [115].

lation, and used it to determine the charged-pion-nucleon coupling constant, with the result $g_{c}^{2} / 4 \pi=13.69 \pm 0.20$.

Perhaps the most ambitious single project within B.3 is the derivation and evaluation of Roy-Steiner equations for pion-nucleon scattering. These are partial-wave dispersion relations, based on hyperbolic dispersion relations and making maximal use of analyticity, unitarity, and crossing symmetry. We have derived a closed system of Roy-Steiner equations [116], where all kernel functions and unitarity relations required for the lowest partial waves have been worked out analytically. In order to suppress the dependence on the high-energy regime, also once- and twice-subtracted versions of these equations were considered, where the subtraction constants were identified with subthreshold parameters. Assuming Mandelstam analyticity we have determined the maximal range of validity of these equations. As a first step towards the solution of the full system, we have cast the equations for the $\pi \pi \rightarrow \bar{N} N$ partial waves into the form of a Muskhelishvili-Omnès problem with finite matching point, which were solved numerically in the single-channel approximation. Some consequences for the spectral functions of the nucleon electromagnetic form factors were also discussed. As an application, we have solved a system of coupled integral equations for the $\pi \pi \rightarrow \bar{N} N$ and $\bar{K} K \rightarrow \bar{N} N$ ( $t$-channel) Swaves in order to update the dispersive analysis of the correction $\Delta_{\sigma}=\sigma\left(2 M_{\pi}^{2}\right)-\sigma_{\pi N}$ in the nucleon scalar form factor, which is needed for the extraction of the pion-nucleon $\sigma$-term from $\pi N$ scattering [117].

The full, self-consistent solution of the Roy-Steiner system of $s$ - and $t$-channel partial waves was documented in detail in Ref. [114]. The result of this analysis is a determination of $s$ - and $t$-channel $\mathrm{S}$ - and P-wave amplitudes at low energies, as well as a consistent set of the subthreshold parameters that act as the subtraction constants of the dispersion relations. S- and P-waves above the matching point of $\sqrt{s}=1.38 \mathrm{GeV}$ are taken as in- put from existing partial-wave analyses, mainly by the GWU/SAID group [118, 119], but also from KarlsruheHelsinki [120, 121]; both also provide necessary input for the inelasticities, as well as for all higher partial waves. Finally, the S-wave scattering lengths extracted from hadronic atoms $[110,111]$ serve as a constraint to the solution.

We regard our work as an update of the rigorous, dispersion-theory-based Karlsruhe-Helsinki analysis, based on modern data input. We have checked throughout that reverting to the input used in that analysis, we reproduce Karlsruhe-Helsinki results, e.g. for the $\sigma$-term, or for the pion-nucleon coupling constant. This is a crucial consistency check of our numerical solution.

One of the most important tasks is a reliable assessment of the errors propagating through the Roy-Steiner solution. We find that for the $s$-channel partial waves at very low energies, the uncertainty is dominated by those in the S-wave scattering lengths (see Fig. 13), while the uncertainties at the matching point dominate there, at "large" energies (for the range of validity of the equations). In the intermediate regime, flat fit minima of the altogether ten subtraction constants that show significant correlations dominate the final error. Resulting $s$-channel S- and Pwave phase shifts including the final error bands are shown in Fig. 14. Similar results for the final $t$-channel solution are given in Ref. [114].

One of the central results is a determination of the pion-nucleon $\sigma$-term [122]. It is given in terms of two subthreshold parameters obtained as solution of the RoySteiner equations, through the Cheng-Dashen low-energy theorem, for which we have improved on the isospinbreaking corrections. The result is

$$
\sigma_{\pi N}=(59.1 \pm 1.9 \pm 3.0) \mathrm{MeV}=(59.1 \pm 3.5) \mathrm{MeV},
$$

where the first error refers to uncertainties from the RoySteiner solution, and the second from those in the lowenergy theorem (mainly due to isospin breaking). The fact that the value in Eq. (1) is significantly larger than the "canonical" value of $45 \mathrm{MeV}$ obtained by Gasser, Leutwyler, and Sainio [123], based on the KarlsruheHelsinki solution, has caused some controversy; not the least because recent lattice simulations, performed at physical pion masses, seem to confirm the lower value. We have shown that the large $\sigma$-term is a direct consequence of the scattering length input, such that there is actually a conflict between lattice results and hadronic atoms spectroscopy [124]. We have suggested that a lattice determination of the $\pi N$ scattering lengths may be a step towards an understanding of this puzzle.

Finally, we have matched the Roy-Steiner results for the subthreshold parameters of pion-nucleon scattering to chiral perturbation theory up to next-to-next-to-nextto-leading order, to extract the pertinent low-energy constants [125]. The convergence of the chiral series is expected to be best at the subthreshold point, far away from all cuts and threshold singularities, such that the resulting LECs should be more reliable than those extracted from fits in the physical region. Also here, we included a com- 

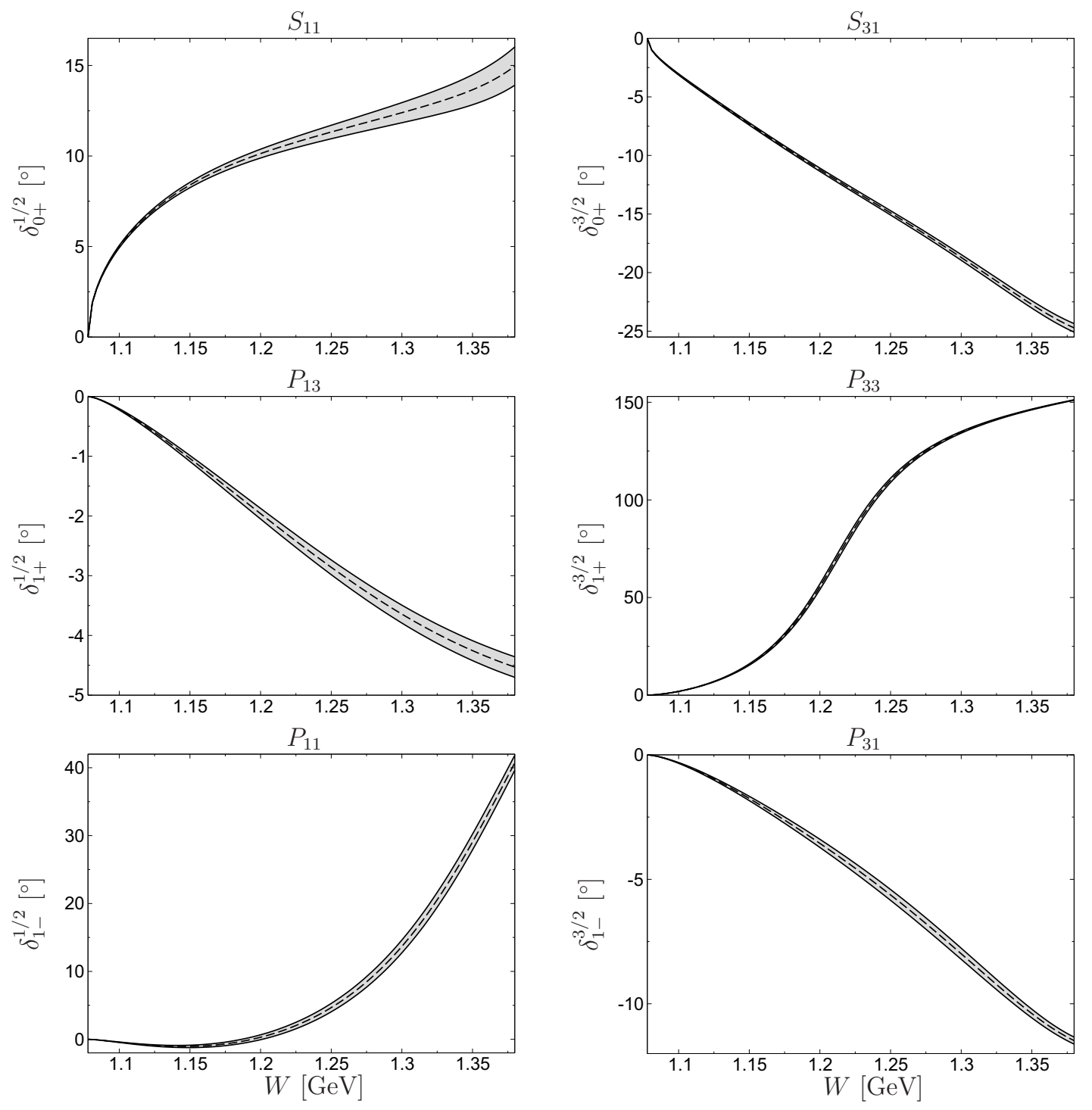

Figure 14. Final errors bands for the $\pi N$ phase shifts. The dashed lines indicate the central curves. Figure taken from Ref. [114].

prehensive analysis of systematic uncertainties and correlations. The convergence of the chiral series, studied by investigating the chiral expansion of threshold parameters up to the same order, turns out to be more problematic than expected, an effect which probably can only partly be explained by the role of the $\Delta$ resonance. Results for the low-energy constants are also presented in the counting scheme usually applied in chiral nuclear effective field theory, where they serve as crucial input to determine the long-range part of the nucleon-nucleon potential as well as three-nucleon forces.

Acknowledgements We thank our collaborators B. Ananthanarayan, Vadim Baru, Véronique Bernard, Moritz Bissegger, Bugra Borasoy, Peter Bruns, Irinel Caprini, Gilberto Colangelo, Ales Cieply, Tobias Dato, Christoph Ditsche, Michael Döring, Evgeny Epelbaum, Matthias Frink, Andreas Fuhrer, Jürg Gasser, Feng-Kun Guo, Hans Werner Hammer, Christoph Hanhart, Johann Haidenbauer, Martin Hoferichter, Hermann Krebs, Siegfried Krewald, Ayse Kucukarslan, Andrzej Kupść, Alexander Kudryavtsev, André Lacour, Timo Lähde, Dean Lee, Vadim
Lensky, Randy Lewis, Stefan Leupold, Valery Lyubovitskij, Maxim Mai, Eike Müller, Franz Niecknig, Robin Nißler, Andreas Nogga, Konstantin Ottnad, Eulogio Oset, José Palomar, Nello Paver, José R. Peláez, Daniel Phillips, Lucas Platter, Judith Plenter, Massimiliano Procura, Udit Raha, Luis Roca, Dino Ruić, Jacobo Ruiz de Elvira, Akaki Rusetsky, Dimitrios Sakkas, Rocco Schiavilla, Martin Schmid, Rebekka Schmidt, Sebastian Schneider, Alexander Sibirtsev, Johan Smejkal, Peter Stoffer, Felix Stollenwerk, Anthony Thomas, Michaela Verbeni, Michele Viviani, Andreas Wirzba, and Chu-Wen Xiao. The work reported here would not have been possible without the support from the Deutsche Forschungsgemeinschaft within the SFB/TR16.

\section{References}

[1] V. Bernard, B. Kubis and U.-G. Meißner, Eur. Phys. J. A 25 (2005) 419 [arXiv:nucl-th/0506023].

[2] A. Schmidt et al., Phys. Rev. Lett. 87 (2001) 232501

[Erratum-ibid. 110 (2013) 039903] [nucl-ex/0105010]. 
[3] J. Gasser, B. Kubis, N. Paver and M. Verbeni, Eur. Phys. J. C 40 (2005) 205 [arXiv:hep-ph/0412130].

[4] E. H. Müller, B. Kubis and U.-G. Meißner, Eur. Phys. J. C 48 (2006) 427 [arXiv:hep-ph/0607151].

[5] B. Kubis, E. H. Müller, J. Gasser and M. Schmid, Eur. Phys. J. C 50 (2007) 557 [arXiv:hep-ph/0611366].

[6] G. Colangelo, J. Gasser, B. Kubis and A. Rusetsky, Phys. Lett. B 638 (2006) 187 [arXiv:hep-ph/0604084].

[7] U.-G. Meißner, G. Müller and S. Steininger, Phys. Lett. B 406 (1997) 154 [Erratum-ibid. B 407 (1997) 454] [hep-ph/9704377].

[8] T. A. Lähde and U.-G. Meißner, Phys. Rev. D 74 (2006) 034021 [arXiv:hep-ph/0606133].

[9] C. Hanhart, B. Kubis and J. R. Peláez, Phys. Rev. D (2007) in print [arXiv:0707.0262 [hep-ph]].

[10] A. Kucukarslan and U.-G. Meißner, Mod. Phys. Lett. A 21 (2006) 1423 [arXiv:hep-ph/0603061].

[11] B. Kubis and R. Lewis, Phys. Rev. C 74 (2006) 015204 [nucl-th/0605006].

[12] M. Frink and U.-G. Meißner, Eur. Phys. J. A 29 (2006) 255 [arXiv:hep-ph/0609256].

[13] J. A. Oller, M. Verbeni and J. Prades, hepph/0701096.

[14] M. Frink and U.-G. Meißner, JHEP 0407, 028 (2004) [arXiv:hep-lat/0404018].

[15] A. Lacour, B. Kubis, and U.-G. Meißner, JHEP 0710 (2007) 083 [arXiv:0708.3957 [hep-ph]].

[16] V. Bernard and U.-G. Meißner, Ann. Rev. Nucl. Part. Sci. 57 (2007) 33 [arXiv:hep-ph/0611231].

[17] U.-G. Meißner, U. Raha and A. Rusetsky, Eur. Phys. J. C 35 (2004) 349 [arXiv:hep-ph/0402261].

[18] G. Beer et al. [DEAR Collaboration], Phys. Rev. Lett. 94 (2005) 212302.

[19] B. Borasoy, U.-G. Meißner and R. Nißler, Phys. Rev. C 74 (2006) 055201 [arXiv:hep-ph/0606108].

[20] U.-G. Meißner, U. Raha and A. Rusetsky, Eur. Phys. J. C 47 (2006) 473 [arXiv:nucl-th/0603029].

[21] L. Roca, C. Hanhart, E. Oset and U.-G. Meißner, Eur. Phys. J. A 27 (2006) 373 [arXiv:nucl-th/0602016].

[22] B. Borasoy, P. C. Bruns, U.-G. Meißner and R. Nißler, Phys. Rev. C 72 (2005) 065201 [arXiv:hep$\mathrm{ph} / 0508307]$.

[23] B. Borasoy, P. C. Bruns, U.-G. Meißner and R. Nißler, Eur. Phys. J. A 34 (2007) 161 [arXiv:0709.3181 [nucl-th]].
[24] A. Sibirtsev, J. Haidenbauer, S. Krewald, U.G. Meißner and A. W. Thomas, Eur. Phys. J. A 31 (2007) 221 [arXiv:hep-ph/0509145].

[25] A. Sibirtsev, U.-G. Meißner and A. W. Thomas, Phys. Rev. D 71 (2005) 094011 [arXiv:hep$\mathrm{ph} / 0503276]$.

[26] A. Sibirtsev, J. Haidenbauer and U.-G. Meißner, Eur. Phys. J. A 27 (2006) 263 [arXiv:nucl-th/0512055].

[27] A. Sibirtsev, H. W. Hammer, U.-G. Meißner and A. W. Thomas, Eur. Phys. J. A 29 (2006) 209 [arXiv:nucl-th/0606044].

[28] B. Borasoy and R. Nißler, Eur. Phys. J. A 26 (2005) 383 [arXiv:hep-ph/0510384].

[29] B. Borasoy, U.-G. Meißner and R. Nißler, Phys. Lett. B 643 (2006) 41 [arXiv:hep-ph/0609010].

[30] B. Borasoy and E. Lipartia, Phys. Rev. D 71 (2005) 014027 [arXiv:hep-ph/0410141].

[31] E. Epelbaum and U.-G. Meißner, Phys. Rev. C 72 (2005) 044001 [arXiv:nucl-th/0502052].

[32] E. Epelbaum, U.-G. Meißner and J. E. Palomar, Phys. Rev. C 71 (2005) 024001 [arXiv:nuclth/0407037].

[33] H. Krebs, E. Epelbaum and U.-G. Meißner, Eur. Phys. J. A 32 (2007) 127-137 [arXiv:nucl-th/0703087].

[34] E. Epelbaum and U.-G. Meißner, Few Body Syst. 54 (2013) 2175 [nucl-th/0609037].

[35] E. Epelbaum, H. W. Hammer, U.-G. Meißner and A. Nogga, Eur. Phys. J. C 48 (2006) 169 [arXiv:hep$\mathrm{ph} / 0602225]$.

[36] H. W. Hammer, D. R. Phillips and L. Platter, Eur. Phys. J. A 32 (2007) 335 [arXiv:0704.3726 [nucl-th]].

[37] B. Borasoy, H. Krebs, D. Lee and U.-G. Meißner, Nucl. Phys. A 768 (2006) 179 [arXiv:nucl-th/0510047].

[38] B. Borasoy, E. Epelbaum, H. Krebs, D. Lee and U.G. Meißner, Eur. Phys. J. A 31 (2007) 105 [arXiv:nuclth/0611087].

[39] L. Platter and H. W. Hammer, Nucl. Phys. A 766 (2006) 132 [arXiv:nucl-th/0509045].

[40] H. W. Hammer and L. Platter, Eur. Phys. J. A 32 (2007) 113 [arXiv:nucl-th/0610105].

[41] M. Viviani et al., Phys Rev. Lett. 99 (2007) 112002 [arXiv:nucl-th/0703051].

[42] V. Lensky, V. Baru, J. Haidenbauer, C. Hanhart, A. E. Kudryavtsev and U.-G. Meißner, Eur. Phys. J. A 26 (2005) 107 [arXiv:nucl-th/0505039]. 
[43] V. Lensky, V. Baru, E. Epelbaum, C. Hanhart, J. Haidenbauer, A. Kudryavtsev and U.-G. Meißner, Eur. Phys. J. A (2007) in print [arXiv:0704.0443 [nuclth]].

[44] B. Kubis, EPJ Web Conf. 3 (2010) 01008 [arXiv:0912.3440 [hep-ph]].

[45] J. R. Batley et al. [NA48/2 Collaboration], Phys. Lett. B 633 (2006) 173 [arXiv:hep-ex/0511056].

[46] M. Bissegger, A. Fuhrer, J. Gasser, B. Kubis and A. Rusetsky, Phys. Lett. B 659 (2008) 576 [arXiv:0710.4456 [hep-ph]].

[47] J. Gasser, V. E. Lyubovitskij and A. Rusetsky, Phys. Rept. 456 (2008) 167 [arXiv:0711.3522 [hep-ph]].

[48] J. R. Batley et al. [Na48/2 Collaboration], Eur. Phys. J. C 64 (2009) 589 [arXiv:0912.2165 [hep-ex]].

[49] M. Bissegger, A. Fuhrer, J. Gasser, B. Kubis and A. Rusetsky, Nucl. Phys. B 806 (2009) 178 [arXiv:0807.0515 [hep-ph]].

[50] B. Kubis and R. Schmidt, Eur. Phys. J. C 70 (2010) 219 [arXiv:1007.1887 [hep-ph]].

[51] J. Gasser, B. Kubis and A. Rusetsky, Nucl. Phys. B 850 (2011) 96 [arXiv:1103.4273 [hep-ph]].

[52] B. Kubis and S. P. Schneider, Eur. Phys. J. C 62 (2009) 511 [arXiv:0904.1320 [hep-ph]].

[53] G. Colangelo, J. Gasser and A. Rusetsky, Eur. Phys. J. C 59 (2009) 777 [arXiv:0811.0775 [hep-ph]].

[54] J. R. Batley et al. [NA48-2 Collaboration], Eur. Phys. J. C 70 (2010) 635.

[55] G. Colangelo, J. Gasser and H. Leutwyler, Phys. Lett. B 488 (2000) 261 [hep-ph/0007112].

[56] C. Ditsche, B. Kubis and U.-G. Meißner, Eur. Phys. J. C 60 (2009) 83 [arXiv:0812.0344 [hep-ph]].

[57] M. Unverzagt et al. [CB-at-MAMI, TAPS, and A2 Collaborations], Eur. Phys. J. A 39 (2009) 169 [arXiv:0812.3324 [hep-ex]].

[58] C. Adolph et al. [WASA-at-COSY Collaboration], Phys. Lett. B 677 (2009) 24 [arXiv:0811.2763 [nuclex]].

[59] S. Prakhov et al. [Crystal Ball at MAMI and A2 Collaborations], Phys. Rev. C 79 (2009) 035204 [arXiv:0812.1999 [hep-ex]].

[60] F. Ambrosino et al. [KLOE Collaboration], Phys. Lett. B 694 (2011) 16 [arXiv:1004.1319 [hep-ex]].

[61] J. Bijnens and K. Ghorbani, JHEP 0711 (2007) 030 [arXiv:0709.0230 [hep-ph]].
[62] S. P. Schneider, B. Kubis and C. Ditsche, JHEP 1102 (2011) 028 [arXiv:1010.3946 [hep-ph]].

[63] K. Nakamura et al. [Particle Data Group Collaboration], J. Phys. G 37 (2010) 075021.

[64] F. Ambrosino et al. [KLOE Collaboration], JHEP 0805 (2008) 006 [arXiv:0801.2642 [hep-ex]].

[65] F.-K. Guo, B. Kubis and A. Wirzba, Phys. Rev. D 85 (2012) 014014 [arXiv:1111.5949 [hep-ph]].

[66] M. Ablikim et al. [BESIII Collaboration], Phys. Rev. Lett. 112 (2014) 251801 [Addendum: ibid. 113 (2014) 039903] [arXiv:1404.0096 [hep-ex]].

[67] K. Ottnad, B. Kubis, U.-G. Meißner and F.-K. Guo, Phys. Lett. B 687 (2010) 42 [arXiv:0911.3981 [hep$\mathrm{ph}]]$.

[68] F. Niecknig, B. Kubis and S. P. Schneider, Eur. Phys. J. C 72 (2012) 2014 [arXiv:1203.2501 [hep-ph]].

[69] A. Aloisio et al. [KLOE Collaboration], Phys. Lett. B 561 (2003) 55 [Erratum-ibid. B 609 (2005) 449] [hepex/0303016].

[70] R. R. Akhmetshin et al., Phys. Lett. B 642 (2006) 203.

[71] S. P. Schneider, B. Kubis and F. Niecknig, Phys. Rev. D 86 (2012) 054013 [arXiv:1206.3098 [hep-ph]].

[72] F. Stollenwerk, C. Hanhart, A. Kupść, U.-G. Meißner and A. Wirzba, Phys. Lett. B 707 (2012) 184 [arXiv:1108.2419 [nucl-th]].

[73] P. Adlarson et al. [WASA-at-COSY Collaboration], Phys. Lett. B 707 (2012) 243 [arXiv:1107.5277 [nuclex]].

[74] D. Babusci et al. [KLOE Collaboration], Phys. Lett. B 718 (2013) 910 [arXiv:1209.4611 [hep-ex]].

[75] M. Mai, P. C. Bruns, B. Kubis and U.-G. Meißner, Phys. Rev. D 80 (2009) 094006 [arXiv:0905.2810 [hep$\mathrm{ph}]]$.

[76] A. Torok, S. R. Beane, W. Detmold, T. C. Luu, K. Orginos, A. Parreno, M. J. Savage and A. Walker-Loud, Phys. Rev. D 81 (2010) 074506 [arXiv:0907.1913 [hep-lat]].

[77] P. C. Bruns, M. Mai and U.-G. Meißner, Phys. Lett. B 697 (2011) 254 [arXiv:1012.2233 [nucl-th]].

[78] D. Ruić, M. Mai and U.-G. Meißner, Phys. Lett. B 704 (2011) 659 [arXiv:1108.4825 [nucl-th]].

[79] M. Mai and U.-G. Meißner, Nucl. Phys. A 900 (2013) 51 [arXiv:1202.2030 [nucl-th]].

[80] E. F. McNicoll et al. [Crystal Ball at MAMI Collaboration], Phys. Rev. C 82 (2010) 035208 [Erratum-ibid. 
C 84 (2011) 029901] [arXiv:1007.0777 [nucl-ex]].

[81] M. Bazzi et al., Phys. Lett. B 704 (2011) 113 [arXiv:1105.3090 [nucl-ex]].

[82] M. Döring, E. Oset and U.-G. Meißner, Eur. Phys. J. A 46 (2010) 315 [arXiv:1003.0097 [nucl-th]].

[83] E. Gutz et al. [CBELSA/TAPS Collaboration], Phys. Lett. B 687, 11 (2010) [arXiv:0912.2632 [nucl-ex]].

[84] M. Döring, E. Oset and D. Strottman, Phys. Rev. C 73, 045209 (2006) [arXiv:nucl-th/0510015].

[85] M. Döring, E. Oset and D. Strottman, Phys. Lett. B 639, 59 (2006) [arXiv:nucl-th/0602055].

[86] G. Colangelo, M. Hoferichter, M. Procura and P. Stoffer, JHEP 1409 (2014) 091 [arXiv:1402.7081 [hep-ph]].

[87] G. Colangelo, M. Hoferichter, B. Kubis, M. Procura and P. Stoffer, Phys. Lett. B 738 (2014) 6 [arXiv:1408.2517 [hep-ph]].

[88] G. Colangelo, M. Hoferichter, M. Procura and P. Stoffer, JHEP 1509 (2015) 074 [arXiv:1506.01386 [hep-ph]].

[89] M. Hoferichter, D. R. Phillips and C. Schat, Eur. Phys. J. C 71 (2011) 1743 [arXiv:1106.4147 [hep-ph]].

[90] M. Hoferichter, B. Kubis and D. Sakkas, Phys. Rev. D 86 (2012) 116009 [arXiv:1210.6793 [hep-ph]].

[91] M. Hoferichter, B. Kubis, S. Leupold, F. Niecknig and S. P. Schneider, Eur. Phys. J. C 74 (2014) 3180 [arXiv:1410.4691 [hep-ph]].

[92] M. N. Achasov et al., Eur. Phys. J. C 12 (2000) 25.

[93] M. N. Achasov et al., Phys. Lett. B 559 (2003) 171 [hep-ex/0302004].

[94] R. R. Akhmetshin et al. [CMD2 Collaboration], Phys. Lett. B 605 (2005) 26 [hep-ex/0409030].

[95] B. Ananthanarayan, I. Caprini and B. Kubis, Eur. Phys. J. C 74 (2014) 3209 [arXiv:1410.6276 [hep-ph]].

[96] I. Caprini, Phys. Rev. D 92 (2015) 014014 [arXiv:1505.05282 [hep-ph]].

[97] C. Hanhart, A. Kupść, U.-G. Meißner, F. Stollenwerk and A. Wirzba, Eur. Phys. J. C 73 (2013) 2668 [Erratum-ibid. C 75 (2015) 242] [arXiv:1307.5654 [hep-ph]].

[98] B. Kubis and J. Plenter, Eur. Phys. J. C 75 (2015) 283 [arXiv:1504.02588 [hep-ph]].

[99] C. W. Xiao, T. Dato, C. Hanhart, B. Kubis, U.G. Meißner and A. Wirzba, arXiv:1509.02194 [hep$\mathrm{ph}]$.
[100] M. Mai and U.-G. Meißner, Eur. Phys. J. A 51 (2015) 3, 30 [arXiv:1411.7884 [hep-ph]].

[101] Y. Ikeda, T. Hyodo and W. Weise, Phys. Lett. B 706 (2011) 63 [arXiv:1109.3005 [nucl-th]].

[102] Z. H. Guo and J. A. Oller, Phys. Rev. C 87 (2013) 035202 [arXiv:1210.3485 [hep-ph]].

[103] A. Ciepý, M. Mai, U.-G. Meißner and J. Smejkal, Nucl. Phys. A 954 (2016) 17 [arXiv:1603.02531 [hep$\mathrm{ph}]]$.

[104] K. Moriya et al. [CLAS Collaboration], Phys. Rev. C 87 (2013) 035206 [arXiv:1301.5000 [nucl-ex]].

[105] K. Moriya et al. [CLAS Collaboration], Phys. Rev. Lett. 112 (2014) 082004 [arXiv:1402.2296 [hep-ex]].

[106] R. J. Hemingway, Nucl. Phys. B 253 (1985) 742.

[107] U.-G. Meißner, T. Hyodo, http://pdg.lbl.gov/2015/ reviews/rpp2015-rev-lam-1405-pole-struct.pdf.

[108] M. Hoferichter, B. Kubis and U.-G. Meißner, Phys. Lett. B 678 (2009) 65 [arXiv:0903.3890 [hep-ph]].

[109] M. Hoferichter, B. Kubis and U.-G. Meißner, Nucl. Phys. A 833 (2010) 18 [arXiv:0909.4390 [hep-ph]].

[110] V. Baru, C. Hanhart, M. Hoferichter, B. Kubis, A. Nogga and D. R. Phillips, Phys. Lett. B 694 (2011) 473 [arXiv:1003.4444 [nucl-th]].

[111] V. Baru, C. Hanhart, M. Hoferichter, B. Kubis, A. Nogga and D. R. Phillips, Nucl. Phys. A 872 (2011) 69 [arXiv:1107.5509 [nucl-th]].

[112] D. Gotta et al., Lect. Notes Phys. 745 (2008) 165.

[113] T. Strauch et al., Eur. Phys. J. A 47 (2011) 88 [arXiv:1011.2415 [nucl-ex]].

[114] M. Hoferichter, J. Ruiz de Elvira, B. Kubis and U.-G. Meißner, Phys. Rept. 625 (2016) 1 [arXiv:1510.06039 [hep-ph]].

[115] M. Hennebach et al., Eur. Phys. J. A 50 (2014) 190 [arXiv:1406.6525 [nucl-ex]].

[116] C. Ditsche, M. Hoferichter, B. Kubis and U.G. Meißner, JHEP 1206 (2012) 043 [arXiv:1203.4758 [hep-ph]].

[117] M. Hoferichter, C. Ditsche, B. Kubis and U.G. Meißner, JHEP 1206 (2012) 063 [arXiv:1204.6251 [hep-ph]].

[118] R. A. Arndt, W. J. Briscoe, I. I. Strakovsky and R. L. Workman, Phys. Rev. C 74 (2006) 045205 [nuclth/0605082].

[119] R. L. Workman, R. A. Arndt, W. J. Briscoe, M. W. Paris and I. I. Strakovsky, Phys. Rev. C 86 (2012) 035202 [arXiv:1204.2277 [hep-ph]]. 
[120] R. Koch and E. Pietarinen, Nucl. Phys. A 336 (1980) 331.

[121] G. Höhler, Pion-Nukleon-Streuung: Methoden und Ergebnisse, in Landolt-Börnstein, 9b2, ed. H. Schopper, Springer Verlag, Berlin, 1983.

[122] M. Hoferichter, J. Ruiz de Elvira, B. Kubis and U.-G. Meißner, Phys. Rev. Lett. 115 (2015) 092301 [arXiv:1506.04142 [hep-ph]].
[123] J. Gasser, H. Leutwyler and M. E. Sainio, Phys. Lett. B 253 (1991) 252.

[124] M. Hoferichter, J. Ruiz de Elvira, B. Kubis and U.-G. Meißner, Phys. Lett. B 760 (2016) 74 [arXiv:1602.07688 [hep-lat]].

[125] M. Hoferichter, J. Ruiz de Elvira, B. Kubis and U.G. Meißner, Phys. Rev. Lett. 115 (2015) no.19, 192301 [arXiv:1507.07552 [nucl-th]]. 